\title{
Recommendations for the management of children with allergic diseases during the epidemic of novel coronavirus pneumonia
}

\author{
Li Feng", Xu Xuefeng", Wu Ping", Guang Kai*, Meiping Lu*, Zhou Wei*, Gary Wong* and Huasong Zeng* \\ Paediatric Allergy, Immunology and Rheumatology Branch, Asia Pacific Association of Medicine and Bio-Immunology (PAIRB-APAMBI), China \\ \#*Contributed equally
}

Experts: Guangzhou Women and Children's Medical Center, Guangzhou Medical University (Zeng Huasong, Li Feng, Xie Yin, Wu Ping, Ren Qi); the First Affiliated Hospital of Guangxi Medical University (Nong Guangmin, Jiang Min); Peking Union Medical College Hospital, Chinese Academy of Medical Sciences \& Peking Union Medical College (Guan Kai, Xu Yingyang, Wan Weilin, Quan Meiying, Cui Le); Peking University Third Hospital (Zhou Wei, Liu Ling); The Children's Hospital of Medical College, Zhejiang University (Lu Meiping, Xu Xuefeng); Peking Children's Hospital Affiliated to Capital Institute of Pediatrics (Qinglong Gu, Yuzhi Chen, Li Sha, Zhong Xuemei, Gao Ying). Beijing Children's Hospital, Capital Medical University, National Center for Children's Health (Jiang Nannan, Tang Lixing, Xing Huan, Xu Wei); Women and Children's Hospital, School of Medicine, Xiamen University (Li Yan); Aviation General Hospital of China Medical University (An Bing); Shenmu City Hospital (Liu Yonglin ); Yuyang Regional People's Hospital (Xue Tao); Beijing Dong Cheng District Maternal and Child Health Hospital (Shi Xuhui); China Agricultural University (Che Huilian); Peking University Hospital (Li Li); Chengde Maternal and Child Health Hospital (Zhang Haibo); Yantai Yuhuangding Hospital (Wang Shuyun); The First Hospital of Jilin University (Yang Sirui, Yin Jianing; The Affiliated Hospital of Qingdao University (Jin Rong); Tianjin Children's Hospital (Hu Jian, Qu Liya); Guangxi Maternal and Child Health Hospital (Qin Min); Shengjing Hospital of China Medical University (Shan Lishen); Guiyang Maternal and Child Health Hospital (Su Shuoishou, Zhan Yi); Boai Hospital of Zhongshan (Huang Dongming, Huang Xiaowen), Bostion Children's Hospital, Harvard University (Peiyu Lee); Prince of Wales Hospital, Chinese University of Hong Kong, Gary Wong, Kiang Wu Hospital, Macau (Lei Cheng, Chen Yan, Chen Rongrong).

\begin{abstract}
During the epidemic period of sars-cov-2, 48 paediatric allergy and clinical immunology experts from mainland China, Hong Kong, Macao and the United States were organized by the Asia Pacific Association of Medicine and Bio-Immunology (APAMBI, http://apambi.com/) had internet meeting. The expert suggestion on the management of children with allergic diseases during the epidemic of Novel coronavirus pneumonia. The clinical characteristics and differential diagnosis of sarscov-2 infection in children with allergy, the prevention of sars-cov-2 infection in children with allergy. During the epidemic period, it is necessary to go back and forth to the special hospital for the management and guidance of children with special treatment, management measures of related issues, treatment of suspected infection of sars-cov-2 in children with allergy, treatment of confirmed infection of sars-cov-2 in children with allergy, suggestions on special medicine, strengthening the management of chronic diseases and diversified diagnosis and treatment services, and establishing the management of chronic diseases during the epidemic period of sars-cov-2 System, online medical treatment and Internet of things system express special drugs, when the disease is aggravated, the guidance of medical treatment. This expert's suggestion has significance Recommendations for the management of children with allergy during the global epidemic of.
\end{abstract}

\section{Short Communication}

Since a new type of coronavirus infection appeared in Wuhan City, Hubei Province in December 2019, the epidemic has spread rapidly throughout the country and other countries. On January 30, 2020, the World Health Organization (WHO) announced that a new outbreak of coronavirus infection was a public health emergency of international concern [1]. In February 2020, WHO named the disease caused by a new type of coronavirus infection as coronavirus disease 2019 (Corona Virus Disease-19, COVID-19) [2]. At the same time, the International Commission for the Classification of Viruses named the virus coronavirus 2 [3] On January 22, 2020, the National Health Council included a new type of coronavirus pneumonia as a B-type infectious disease and prevented it in accordance with A-type infectious diseases [4].
The new coronavirus is sensitive to UV, temperature, $56^{\circ} \mathrm{C}$ for $30 \mathrm{~min}$, ether, $75 \%$ ethanol, chlorine disinfectant, peracetic acid and chloroform and other fat-soluble agents can effectively inactivate the virus, chlorine has been determined not to be effective inactivated. The virus is highly contagious, with epidemiological studies suggesting a general susceptibility to SARS-CoV-2, and a growing number of confirmed cases in children and infants, mostly due to a new type of

${ }^{*}$ Correspondence to: Huasong Zeng, MD, PhD, Paediatric Allergy, Immunology and Rheumatology Branch, Asia Pacific Association of Medicine and BioImmunology (PAIRB-APAMBI), China, E-mail: huasongxuqing@163.com

Key words: Management, children, allergy, epidemic, sars-cov-2

Received: March 18, 2020; Accepted: March 23, 2020; Published: March 26, 2020 
coronavirus from parents or chaperones, so it's common in families with clustered cases with critical cases reported [5]. The youngest child was a new-born 36 hours after birth [6].

The incidence of allergic diseases in children is increasing year by year worldwide [7]. Common allergic diseases in children include allergen-induced asthma, rhinitis, atopic eczema, conjunctivitis, food allergies, insect allergies and anaphylactic shock. Children with allergic diseases may be more susceptible to SARS-CoV-2 because of disorders in their own immune system and long-term use of certain drugs. The incidence of allergic rhinitis, allergic conjunctivitis and allergic asthma increased with the warming of spring climate [8]. The incidence of allergic diseases in children is also influenced by many factors, such as infection and psychology. During the COVID-19 epidemic period, children mostly have home activities. Indoor triggers, anxiety and failure to follow up on time have brought difficulties to the management of allergic diseases in children. To avoid COVID-19 in children with allergic diseases and to strengthen the management of primary underlying diseases, follow the diagnosis and treatment program issued by the National Health and Health Council [9]. According to the clinical characteristics of children with COVID-19, some suggestions for prevention and treatment of children with allergic diseases under COVID-19 were put forward for clinical reference.

\section{Home and Out Protection Guide}

SARS-CoV-2 is transmitted mainly through spreading airborne and contact. Prevention of SARS-CoV-2 infection is important, and prevention is more important than treatment [10]. Most children with SARS-Cov-2 infection are caused by close family contact. Children with allergic diseases, parents or relatives should be protected against:

\section{Learn more about cough etiquette and hand hygiene}

Avoid coughing or sneezing in public (or without tissue) as much as possible. Cover your mouth and nose with a tissue and hands. If you can't find a tissue temporarily, bend your elbow and cover your mouth and nose. Wash hands with running water, strictly enforce hand hygiene, according to the six-part hand washing method [11]. Hand hygiene includes washing your hands before and after meals, after going home, after contact with garbage, after touching animals, before and after wearing a mask. Try not to touch mouth, nose and eyes before washing your hands.

\section{Avoid going to the crowded or poorly ventilated places as far as possible}

Wear a mask for going out and travelling on public transport and avoid using closed public transport as far as possible. It is recommended to use a child-specific mask in accordance with the national standards. Family members who contact with COVID-19 suspected or diagnosed patients should not contact with children until they are strictly isolated for at least 14 days.

\section{During the COVID-19 epidemic, most of the children remained indoors}

The influence of indoor environment on children with allergic diseases was significantly increased. In door allergens are one of the important factors that induce the onset of allergic diseases, among which dust mites are the most common inhaled allergens in the room, and are closely related to the acute onset of allergic diseases in children (such as: asthma, allergic rhinitis, allergic conjunctivitis, etc.) Room environment should be cool, ventilated, clean, humidity $30 \%$ to $50 \%$ appropriate, recommended to clean the room with wet mop and rag, do not keep pets with hair, avoid house dust mites, dust, animal hair and other human allergen inhalation; maintain the circulation of indoor air, generally can open windows to change air 2-3 times a day, not less than 30 minutes each time, different rooms can take turns. It is best to move the child into another room when the room is ventilated. Keep warm and avoid catching cold when ventilating.

\section{Use of disinfectants}

Exposure to physical and chemical factors such as disinfectants can induce recurrence of allergic diseases in children. Do not use high concentrations of chlorine-containing disinfectants, and do not mix with other types of disinfectants or cleaners to avoid the risk of a large amount of chlorine release; use chlorine-containing disinfectants for disinfection in normal homes up to once a day; open all windows to ventilate during the use of disinfectants; if the child is highly sensitive to disinfectants, replace it with ultraviolet radiation, recommend disinfection once a day for not less than $30 \mathrm{~min}$, or air disinfection (fixed air dis infector), once a day for $30 \mathrm{~min}$.

\section{Monitoring of children and all family members}

Observe fever, runny nose, cough, diarrhoea and other symptoms, and make relevant records.

Children with exposed to suspected or diagnosed cases of new coronary pneumonia

Child exposed to suspected/diagnosed COVID-19 cases, currently asymptomatic

- In addition to daily protection, children should be isolated at home to observe, as far as possible do not go out [12].

- Medical observation: It is recommended to monitor the body at least every 6 to 8 hours. Observe the respiratory symptoms with or without COVID-19 such as cough, sore throat, shortness of breath, gastrointestinal symptoms such as diarrhoea, nausea, vomiting, and no headache, fatigue, lethargy, mental retardation, etc., and record them in time.

- Reminds the child to wear a medical mask or a N95 mask throughout the course of the visit, and truthfully informs the medical staff of the contact history of the diagnosis and suspected cases of COVID-19 or the contact history of the patients with cluster disease.

- Warns family members to strengthen their personal protection by wearing medical masks and limiting the number of carers, reducing or avoiding changes in carers.

- Increase the ventilation and disinfection of the home environment.

In cases where fever, respiratory tract or digestive tract symptoms described above, or in the course of observation at home, may occur after exposure to suspected/diagnosed cases of COVID-19.

\section{Protection and monitoring of children with suspected or diagnosed cases of new coronary pneumonia}

For children with suspected or diagnosed SARS-Cov-2 infection, the local CDC should be notified immediately and transferred to designated institutions with effective isolation and protection conditions. The suspected cases should be treated in a single isolation unit. The confirmed cases can be treated in the same ward. Specific trans-shipment programmes refer to the National Health Council's Programme of Work on the Transport of Pneumonia Cases with 
New Coronary Virus Infections (Pilot) [13]. The symptoms of upper respiratory tract infection such as nasal congestion, runny nose and sore throat may be present in children with COVID-19 [14]. The number of confirmed cases in children with milder clinical symptoms has been reported. However, in children with immune disorders caused by allergic diseases, virus virulence may be heavier, longer, and virus clearance may be prolonged. In addition, the virus may activate the immune system, causing recurrent outbreaks of allergic diseases, making clinical treatment more difficult and more complex. Therefore, on the basis of symptomatic treatment, we should actively prevent and treat complications, treat basic diseases, prevent secondary infections, and carry out organ function support in time.

\section{Treatment of children with allergic diseases during the 4 . COVID-19 epidemic}

- During the current period of COVID-19 epidemic, parents should observe the symptoms of children with allergic diseases closely and contact their attending doctors as much as possible to obtain the correct treatment. Mild seizures can be dealt with in the family, moderate and severe attacks need timely hospital visits.

- If the condition is well controlled, it is recommended to maintain the current treatment; if the condition is repeated or aggravated, the medication may be adjusted according to the symptoms, and the medication for symptomatic treatment may be appropriately increased.

- Patients with severe conditions will be treated with glucocorticoids. Current guidelines suggest using glucocorticoids in a short period of time (3-5 days), as appropriate, based on the extent of dyspnoea and the progress of chest imaging, with a recommended dose of no more than 1-2 mg/(kg.d) of methylprednisolone [15]. Children with long-term use of hormones who are not suitable for continued glucocorticoid therapy and cannot be discontinued abruptly should be gradually discontinued or maintained at a daily low dose of prednisone $<0.5 \mathrm{mg} / \mathrm{kg}$. In case of epidemic, try to avoid atomization treatment in hospital.

- Allergen-specific immunotherapy (AIT): There are two prescriptions for AIT, namely sublingual administration (SLIT) and subcutaneous injection (SCIT). During the COVID-19 epidemics, you can continue sublingual therapy at home under the guidance of a doctor. In the non-peak phase of the epidemic, children with allergic diseases can normally carry out SCIT in the hospital at the prescribed time; in the severe area of the epidemic or the peak phase of the epidemic, in order to reduce the risk of virus infection from the hospital to and from the hospital and after the injection, four weeks before the initial dose phase of the SCIT, the treatment is recommended to stop; in the initial phase of 4 weeks, the latter can be postponed for 2 weeks, then the dose can be adjusted according to the situation; in the maintenance phase, the injection can be postponed after 2 weeks, if the injection is delayed for more than 2 weeks, the dose should be adjusted again according to the delayed condition; in principle, it is not recommended for more than 8 weeks, but more than 8 weeks, the initial injection phase needs to be readjusted.

- Biological agents: At present, the domestic children aged 6 and over asthma patients are approved to use the Omazhu single. Patients with severe active infections should temporarily avoid the use of biological agents until the infection subsides, and those with bacterial infections need to be properly treated with effective antibiotics. In the off-peak stage of the epidemic, the injection treatment can be carried out in the hospital normally according to the prescribed time; in the severe area of the epidemic or the peak stage of the epidemic, it is recommended to postpone the injection of omazumab as appropriate, while maintaining the dosage or increase of the original controlled drug as appropriate.

\section{Strengthening Clinical Services}

At present, many hospitals in China have opened Internet hospitals, which have a series of functions, such as voice and graphic consultation, follow-up, electronic prescription and drug distribution. Parents can communicate with doctors through online hospitals to minimize the number of visits to hospitals. Children are encouraged to conduct laboratory tests or to purchase medicines in the nearest place under the guidance of a doctor. When visiting a hospital, children and parents should be protected, especially from cross-infection by wearing a mask throughout the hospital.

Wait.

\section{References}

1. https://www.who.int/news-room/events/detail/2020/01/30/default-calendar/ international-health-regulations-emergency-committee-on-novel-coronavirus-in-china

2. https://www.who.int/emergencies/diseases/novel-coron avirus-2019/situation-reports/

3. Gorbalenya AE, Baker SC, Baric RS, et al. (2020) Severe acute respiratory syndrome -related coronavirus -The specs and its viruses, a statement of the Coronavirus Study Group. BioRxiv 02: 937862.

4. http://www.nhc.gov.cn/jkj/s7916/202001/44a3b8245e8049d2837a4f27529cd386. shtml

5. Zhu N, Zhang D, Wang W, Li X, Yang B, et al. (2020) A novel coronavirus from patients with pneumonia in China, 2019. N Engl J Med. Epubhead of print.

6. Gorbalenya AE, Baker SC, Baric RS, et al. (2020) Severe acute respiratory syndrome -related coronavirus: The specifics and its viruses- a statement of the Coronavirus Study Group. BioRxiv. Epubhead of print.

7. Gaugris S, Sazonov-Kocevar V, Thomas M (2006) Burden of concomitant allergic rhinitis in adults with asthma. J Asthma. 43: 20-23. [Crossref]

8. Ma TT, Zhuang Y, Gong HY, Yii AC, Wang XY, et al. (2017) Predictive value of respiratory symptoms for the diagnosis of pollen-induced seasonal asthma among children and adults in Inner Mongolia. Ther Clin Risk Manag. 13: 967-974. [Crossref]

9. http://www.nhc.gov.cn/yzygj/s7653p/202002/8334a8326dd94d329df351d7da8aefc2. shtml

10. Xinhua L, Fu G. (2020) Guidelines for Public Protection against Pneumonia with New Coronary Virus Infection [M]. Beijing: People's Health Publishing House.

11. National Health and Health Commission (2019) Hand hygiene standards for medical personnel.

12. Mo W, Jianhua Z, Qian S, Jianhua M, Yuhong T, et al. (2020) Recommendations for prevention and control of novel coronavirus infection in children with chronic kidney disease. Chin J Nephrol 36: 89-93.

13. http://www.gov.cn/zhengce/zhengceku/2020-01/29/content_5472894.html

14. Yi J, Runming J, Zheng Yuejie, et al. (2020) Expert consensus on the diagnosis, treatment and prevention of new type of coronavirus infection in children. Chinese Journal of Practical Pediatrics 35: 81-85. [Crossref]

15. Dongchi Z, Runming J, Zhisheng L (2020) Recommendations for the diagnosis and treatment of new coronavirus infection in children in Hubei province. Chinese Journal of Contemporary Pediatrics 22: 96-99.

Copyright: (C2020 Feng L. This is an open-access article distributed under the terms of the Creative Commons Attribution License, which permits unrestricted use, distribution, and reproduction in any medium, provided the original author and source are credited. 